\title{
Improving the Sensitivity and Specificity of Protein Homology Search by Incorporating Predicted Secondary Structures
}

\author{
Bin Ma, Lieyu Wu, and Kaizhong Zhang \\ Department of Computer Science, University of Western Ontario, \\ London, ON N6A 5B7, Canada \\ \{bma, lwu7, kzhang\}@csd.uwo.ca
}

\begin{abstract}
In this paper, we improve the homology search performance by the combination of the predicted protein secondary structures and protein sequences. Previous research suggested that the straightforward combination of predicted secondary structures did not improve the homology search performance, mostly because the errors in the structure prediction. We solved this problem by taking into account the confidence scores output by the prediction programs.
\end{abstract}

\section{Introduction}

Proteins are essential to all living beings. They are involved in almost every cell function as enzymes and act as structural material of cells. The primary structure of a protein is a sequence of amino acids. The similarity at the sequence level of two proteins is often used to detect protein homology in practice. Tremendous amount of research has been done in the comparison of protein sequences.

For measuring the similarities between pairs of amino acids, amino acid score matrices have been developed, the most popular ones being the BLOSUM matrices [10] and the PAM matrices [9. Once a score matrix is chosen, the SmithWaterman algorithm [18] can be used to compute the the optimal sequence alignment of the two protein sequences. The score of the alignment is equal to the sum of the scores between pairs of amino acids at every position of the alignment.

Usually, a protein sequence is compared with every protein sequence in a protein database to find possible homologs. Protein databases are typically large. As a result, it would take too much time to run the Smith-Waterman algorithm to compare the query protein with every protein in the databases. To address this speed issue, researchers have developed heuristic algorithms to quickly identify the possible targets and run Smith-Waterman-like algorithm only on the possible targets. The most famous software program utilizing such a strategy is BLAST [1,2]. The other popular programs include FASTA [14] and PatternHunter [15, 13.

Besides the speed issue, there are two other important issues, the sensitivity and the specificity, to evaluate the quality of a homology search method. 
Sensitivity measures the ability of the programs to find all of the real homologies. The loss of sensitivity comes from two reasons. The first is that programs like BLAST, FASTA and PatternHunter all use heuristic methods that sacrifice the sensitivity to gain speed. The recent PatternHunter II paper uses multiple spaced seeds to achieve close to Smith-Waterman sensitivity with much faster speed [13]. Therefore, this reason of losing sensitivity has been mostly solved.

However, the second reason of losing sensitivity is that the score defined purely by protein sequence alignment may not be able to recognize the homologies at all. Researchers have noticed that when the sequence identity between known protein and their homologs falls below a threshold of around $20 \%$, sequence alignment are not reliable for finding the homologs [3. This reason of losing sensitivity cannot be solved by any methods that are purely based on sequence comparison.

Specificity measures the ability of the programs of excluding the faked homologies. Because the score defined by purely sequence comparisons is not a very accurate measure of homology, if we set the score threshold low in order to get more real homologs, we end up with picking up many faked homologs with certain sequence similarities.

The secondary structures of proteins have been used to help the homology finding [4,21. The theory is that protein structures are well known to be much more conserved than the protein sequences during the evolution. However, because predicted secondary structures contain errors, the straightforward use of the protein secondary structure did not give any improvement [21] on the performance. Hence, either further criteria were needed to determine the real homologs after the initial selection using secondary structures [4], or the alignments need to be done multiple times with different settings of weights on the sequences and structures [21], which reduces the running speed by multiple folds.

Many computational methods such as those in [8, 17, 12] have been developed for predicting the secondary structure from the amino acid sequence of a protein. Some of them, including PSIPRED [12, give a confidence score to each position of the prediction. In this paper we propose to utilize this confidence score in homology searches. We will show that this improves the homology search sensitivity and specificity significantly by extensive test on some data, as well as prove some nice mathematical properties of the proposed scoring methods.

The contributions of this paper include:

(1) New scoring methods that utilize the predicted secondary structures and the prediction confidence;

(2) Software that uses the new scoring method to do homology search.

\section{A New Scoring Method for Homology Search}

\subsection{Protein Secondary Structure}

The secondary structure of a protein specifies the local spacial arrangements of the protein sequences. Three general types of local arrangements (secondary 
structure units) can be formed. They are the $\alpha$ helix $(\mathrm{H}), \beta$ sheet $(\mathrm{E})$ and coil $(\mathrm{C})$. In the representation of a secondary structure, each amino acid of the protein is labeled with one of the three letters, H, E and C, indicating the amino acid is involved in a unit of the corresponding type. For example, the following is a segment of a protein sequence with its secondary structure:

\section{Amino Acid Sequence: \\ . . . VLSATDKSNVKAAWGKVGG . . . Secondary Structure: \\ ... ССCHНHНHНHНHНHHHHCC. . .}

The amino acid and its corresponding secondary structure label is called an aa/ss pair (amino acid/secondary structure pair) in the paper. Consequently, the sequence and secondary structure together can be regarded as a sequence of aa/ss pairs.

Given an amino acid sequence, a secondary structure prediction program can be used to predict the secondary structure. Many prediction programs including PSIPRED [12] gives a confidence score to the prediction at each position. The confidence score of PSIPRED ranges from 0 to 9 . For example, the following is a segment of PSIPRED's output when using a protein sequence as input:

... VLSATDKSNVKAAWGKVGG . . .

. . . СССНHНHНHНHНHНHНHCC . . .

. . 98899999999999744301...

Although PSIPRED outputs a number between 0 to 9 as its confidence score at each position, for convenience, later on we divide this score by 10 to normalize it to be a value between 0 and 1 .

\subsection{The Proposed Scoring Functions}

In [21, the Smith-Waterman algorithm is utilized to align two sequences of aa/ss pairs. And the optimal local alignment score is used as the similarity score between two proteins. More specifically, the author first computed a secondary structure score matrix, $M^{s s}$, by using a statistical method similar to [10. The $M^{s s}$ given in 21] is shown in Table 1

Then an amino acid score matrix, denoted by $M^{a a}$, is selected to measure the similarity between amino acids. $M^{a a}$ can be set to be one of the BLOSUM matrices or PAM matrices.

Let $\alpha$ and $\beta$ be the weighted importance of the amino acid and secondary structure in the alignment. Let $\langle a, s\rangle$ and $\left\langle a^{\prime}, s^{\prime}\right\rangle$ be two aa/ss pairs. The similarity score of the two pairs is defined by

$$
M\left(\langle a, s\rangle,\left\langle a^{\prime}, s^{\prime}\right\rangle\right)=\alpha \times M^{a a}\left(a, a^{\prime}\right)+\beta \times M^{s s}\left(s, s^{\prime}\right)
$$

Table 1. The structure score matrix used by Wallqvist et. al. 2000

\begin{tabular}{|r|rrr|}
\hline & $\mathrm{H}$ & $\mathrm{E}$ & $\mathrm{C}$ \\
\hline $\mathrm{H}$ & 2 & -15 & -4 \\
$\mathrm{E}$ & -15 & 4 & -4 \\
$\mathrm{C}$ & -4 & -4 & 2 \\
\hline
\end{tabular}


Let $g_{o}$ be the gap open penalty, and $g_{e}$ be the gap extension penalty. The similarity score of two proteins is defined in 21 by the optimal local alignment score of the two sequences of aa/ss pairs.

In 21], $(\alpha, \beta)$ is set to be $(1,0),(0.9,0.1), \ldots,(0,1)$, respectively. The study of 21] demonstrated that if the true secondary structures of the proteins are used, then the performance is maximized for $(\alpha, \beta)=(0.5,0.5)$, i.e., when putting equal weight at the sequence and secondary structure. However, if the predicted secondary structures are used, then the performance is the best for $(\alpha, \beta)=$ $(1,0)$, i.e., when not using secondary structures at all.

This is somewhat discouraging for the use of predicted secondary structures. The authors of 21] then proposed to do eleven different alignments for the eleven different values of $(\alpha, \beta)$ in a row, and use the cumulative homology search results. This indeed showed some degree of improvement when the predicted secondary structures are used. However, this immediately increases the running time by a factor of 10 , which is usually not acceptable.

The results in 21] suggest that the true secondary structures can improve the homology search performance. However, the errors in the predicted secondary structures make them not much useful. Therefore, we propose to use the confidence scores output by the secondary structure prediction programs to distinguish the positions with more accurate predictions from the positions with more errors; and put more weight to the more accurately predicted positions in the score computation.

More formally, let $\langle a, s\rangle$ and $\left\langle a^{\prime}, s^{\prime}\right\rangle$ be two aa/ss pairs. Let $p$ and $q$ be the confidence scores of the predictions at the two pairs, respectively. $0 \leq p, q \leq 1$. Then the similarity score of the two pairs is defined by

$$
M\left(\langle a, s\rangle,\left\langle a^{\prime}, s^{\prime}\right\rangle\right)=M^{a a}\left(a, a^{\prime}\right)+p \times q \times M^{s s}\left(s, s^{\prime}\right)
$$

Once the scores between aa/ss pairs are defined by (2), for any given gap open and gap extension penalty, the standard optimal local alignment score of the two aa/ss sequences is used to be the similarity score between two proteins.

We note that there are ways other than (2) to define the aa/ss score, as follows

$$
M\left(\langle a, s\rangle,\left\langle a^{\prime}, s^{\prime}\right\rangle\right)=M^{a a}\left(a, a^{\prime}\right)+\min \{p, q\} \times M^{s s}\left(s, s^{\prime}\right)
$$

and

$$
M\left(\langle a, s\rangle,\left\langle a^{\prime}, s^{\prime}\right\rangle\right)=M^{a a}\left(a, a^{\prime}\right)+(\min \{p, q\})^{2} \times M^{s s}\left(s, s^{\prime}\right)
$$

The aa/ss score defined in (2) is very intuitive. However, in the next section we will show that the scores defined by (3) and (4) have important properties that (2) does not have.

\subsection{The Strong Similarity Measure}

In homology searches, similarity measures are usually used, where identical sequences have the maximized similarity score. The advantage of using similarity measure is that the optimal local alignment can be nontrivially defined. This is 
not the case for edit distance because the edit distance [6] of two empty strings give us the optimal value 0 .

However, the disadvantage of similarity measure is that there is no Triangular Inequality, which can be very useful for building efficient database indexes such as M-tree [7. Fortunately, researchers observed that most BLOSUM matrices satisfy the following two properties [20].

$$
\begin{gathered}
M(x, y) \leq \min \{M(x, x), M(y, y)\} \\
M(x, y)+M(y, z) \leq M(x, z)+M(y, y)
\end{gathered}
$$

The two properties can be regarded as the equivalence of Triangular Inequality in the similarity measure. Once these properties are satisfied, in this paper we call the similarity measure a strong similarity measure.

The two properties of a strong similarity measure have been exploited to index protein databases for faster homology searches in previous research such as [20, 11, 19]. It is shown in [20,11, 19] that if the score matrix is a strong similarity measure, then the optimal local alignment score based on that score matrix is also a strong similarity measure. Therefore, it is desirable to have the score matrices defined in Section 2.2 be strong similarity measures.

Lemma 1. Let $M(\cdot, \cdot)$ be a strong similarity measure. For any object $x, 0 \leq$ $p_{x} \leq$ be a real number. Then the measure $M^{\prime}(x, y)=M(x, y) \times \min \left\{p_{x}, p_{y}\right\}$ is also a strong similarity measure.

Proof. Proof will be provided in the full version of this extended abstract.

Lemma 2. Let $M(\cdot, \cdot)$ be a strong similarity measure. For any object $x, 0 \leq$ $p_{x} \leq$ be a real number. Then the measure $M^{\prime}(x, y)=M(x, y) \times\left(\min \left\{p_{x}, p_{y}\right\}\right)^{2}$ is also a strong similarity measure.

Proof. From Lemma 1, we know that $M(x, y) \times\left(\min \left\{p_{x}, p_{y}\right\}\right)$ is a strong similarity measure. By using Lemma 1 again, we know that $\left[M(x, y) \times\left(\min \left\{p_{x}, p_{y}\right\}\right)\right] \times$ $\left(\min \left\{p_{x}, p_{y}\right\}\right)$ is a strong similarity measure.

Lemma 3. If $M_{1}$ and $M_{2}$ are strong similarity measures, then $M_{1}+M_{2}$ are strong similarity measure.

Proof. Trivial.

Lemmas 1, 2] and 3 show that if the amino acid matrix, $M^{a a}$, and the secondary structure matrix, $M^{s s}$, are strong similarity measure, then the scores defined by (3) and (4) are also strong similarity measure. On the contrary, we show in the following that the score defined by (2) may be not.

For conflicting the property of (5), suppose that $M(x, y)=1, M(x, x)=2$, $p_{x}=0.1$, and $p_{y}=0.9$. Then $0.09=M(x, y) \times p_{x} \times p_{y}>M(x, x) \times p_{x} \times p_{x}=0.02$

For conflicting the property of ([6), suppose that $M(x, y)=-1, M(y, z)=-1$, $M(x, z)=-1, M(y, y)=1, p_{x}=0.9, p_{y}=0.1$ and $p_{z}=0.9$. Then $-0.18=$ $M(x, y) \times p_{x} \times p_{y}+M(y, z) \times p_{y} \times p_{z}>M(x, z) \times p_{x} \times p_{z}+M(y, y) \times p_{y} \times p_{y}=-0.8$. 


\section{Experiments}

We implemented the Smith-Waterman algorithm by using the score schemes defined by (2), (3), and (4), respectively. For comparison purpose, we also implemented the Smith-Waterman algorithm by using the score scheme defined in the following.

$$
M\left(\langle a, s\rangle,\left\langle a^{\prime}, s^{\prime}\right\rangle\right)=M^{a a}\left(a, a^{\prime}\right)+M^{s s}\left(s, s^{\prime}\right) .
$$

In (17), the predicted secondary structure is used but the confidence scores are not. In all the implementations, the BLOSUM62 matrix is used as the amino acid matrix $M^{a a}$ and the matrix in Table 1 is used as the secondary structure matrix $M^{s s}$. The gap open penalty $g_{o}=-12$ and the gap extension penalty $g_{e}=-2$. We used the PSIPRED [12] program to predict the secondary structures of all proteins that we deal with. The version of PSIPRED we used is 2.3.

The database we used to search in is the release 39 (December 31, 1993) of the PIR database [5], which was downloaded from http://pir.georgetown.edu/. The reason we use this version of the database is to be consistent with [16. Also, we used the same 54 protein superfamilies as used in [16.

For each superfamily, a representative protein was given in [16]. We use that representative protein as query to search the whole PIR database. Suppose the superfamily has size $K$, and the top $K$ sequences given by our search has $K^{\prime}$ sequences in the superfamily. Then $K-K^{\prime}$ sequences are errors. And the ratio

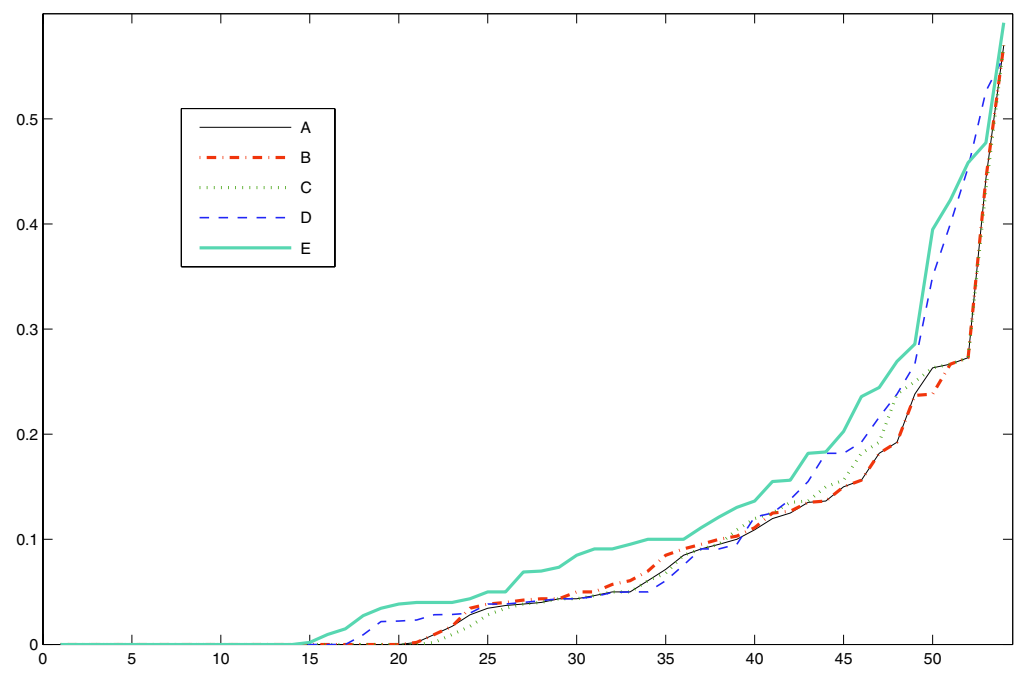

Fig. 1. The EER curve of the five scoring functions on the 54 protein families. A) sequence + weighted structure score as in(2); B) sequence + weighted structure score as in (3); C) sequence + weighted structure score as in(4); D) sequence score only; E) sequence + structure score as in (7) 
$\frac{K-K^{\prime}}{K}$ is called the equal error rate (ERR) of the searching method at that superfamily. We then sort the 54 ERR values and plot a ERR curve in Figure 1

From Figure 1 1 one can conclude that simply adding the structure score based on the predicted secondary structure will make the performance worse (curve $\mathrm{E})$. This is consistent with the observation in 21. However, by accounting for the confidences predicted by the secondary structure prediction program, the performance is much better (curves $\mathrm{A}, \mathrm{B}$ and $\mathrm{C}$ ).

\section{Discussion}

We proposed three new formulae, (2), (3), and (4) to combine the amino acid score and secondary structure score in homology search. By using PSIPRED software to predict the secondary structures of both the query protein sequence and the database protein sequences, the combined score gave significantly better sensitivity and specificity than the ordinary searching method which uses only the amino acid sequences. Our research result is surprising because previous research [21] suggested that combining the predicted secondary structures (instead of the true secondary structures) was not better than using sequences only. The difference between our research and the previous ones is that we take into account the confidence scores output by the secondary structure prediction programs.

When the amino acid matrix and the secondary structure matrix are both strong similarity measures, some of the formulae we used ((3) , and (44)) give us strong similarity measures.

As future work, the gap open and gap extension penalties need to be tuned for different amino acid matrices and secondary structure matrices. The software programs for the experiments will be made available at http://bif.csd.uwo.ca/.

\section{Acknowledgment}

This research was undertaken, in part, thanks to funding from NSERC, PREA, and the Canada Research Chairs Program. We thank Dr. Dong Xu for his recommendation of the PSIPRED secondary structure prediction program.

\section{References}

1. S. F. Altschul, W. Gish, W. Miller, E. W. Myers, and D. J. Lipman. Basic local alignment search tool. Journal of Molecular Biology, 215(3):403-410, 1990.

2. S. F. Altschul, T. L. Madden, A. A. Schaffer, J. Zhang, Z. Zhang, W. Miller, and D. J. Lipman. Gapped BLAST and PSI-BLAST: a new generation of protein database search programs. Nucleic Acids Research, 25(17):3389-3392, 1997.

3. T. K. Attwood and D. J. Parry-Smith. Introduction to Bioinformatics. Prentice Hall, 1999. 
4. R. Auroroa and G. Rose. Seeking an ancient enzyme in methanococcus jannaschii using orf, a program based on predicted secondary structure comparisons. Proc. Natl. Acad. Sci. USA, 95:2818-2823, 1998.

5. G. D. Barker and W. L. Hunt. Pir-international protein sequence database. Methods Enzymol, 266:41-59, 1996.

6. D. Brown, M. Li, and B. Ma. The homology search methods. In L. Wong, editor, The Practical Bioinformatician. World Scientific, 2004.

7. P. Ciaacia, M. Patella, and P. Zezula. M-tree: An efficient access method for similarity search in metric spaces. In Proceedings of the 23rd International Conference on Very Large Database, pages 15-26, 1997.

8. J. A. Cuff and G. J. Barton. Evaluation and improvement of multiple sequence methods for protein secondary structure prediction. PROTEINS: Structure, Function and Genetics, 34:508-519, 1999.

9. M. O. Dayhoff, R. M. Schwartz, and B. C. Orcutt. A model of evolutionary change in proteins. Atlas of Prot. Seq. and Struct., 5:345-352, 1978.

10. S. Henikoff and J. G. Henikoff. Amino acid substitution matrices from protein blocks. Proc. Natl. Acad. Sci. USA, 89:10915-10919, 1992.

11. M. Itoh, S. Goto, T. Akutsu, and M. Kanehisa. Fast and accurate database homology search using upper bounds of local alignment. Bioinformatics, 2004. To appear.

12. D. T. Jones. Protein secondary structure prediction based on position-specific scoring matrices. Journal of Molecular Biology, 292:195-202, 1999.

13. M. Li, B. Ma, D. Kisman, and J. Tromp. PatternHunter II: Highly sensitive and fast homology search. Journal of Bioinformatics and Computational Biology, 2(3):417-440, 2004.

14. D. Lipman and W. Pearson. Rapid and sensitive protein similarity searches. Science, 227, 1985.

15. B. Ma, J. Tromp, and M. Li. PatternHunter: faster and more sensitive homology search. Bioinformatics, 18(3):440-445, March 2002.

16. W. R. Pearson. Comparison of methods for searching protein sequence databases. Protein Science, 4:1145-1160, 1995.

17. B. Rost and C. Sander. Prediction of protein secondary structure at better than 70\% accuracy. Journal of Molecular Biology, 232:584-599, 1993.

18. T. Smith and M. Waterman. Identification of common molecular subsequences. J.Mol.Biol, 147:195-197, 1981.

19. P. Spiro and N. Macura. A local alignment metric for accelerating biosequence database search. Journal of Computational Biology, 11:61-82.

20. A. Stojmirović and V. Pestov. Indexing schemes for similarity search in datasets of short protein fragments. Technical report, 2003. ArXiv e-print DS/0309005.

21. A. Wallqvist, Y. Fukunishi, L. R. Murphy, A. Fadel, and R. M. Levy. Iterative sequence/secondary structure search for protein homologs: comparison with amino acid sequence alignments and application to fold recognition in genome databases. Bioinformatics, 16(11):988-1002, 2000. 